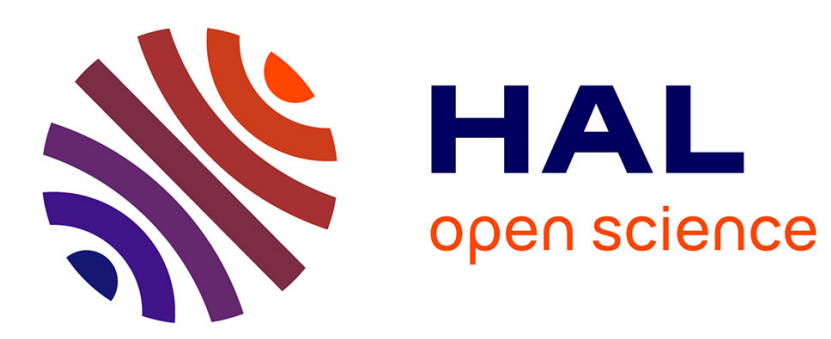

\title{
Spectrum of 1D quasicrystals near the periodic chain
}

Clément Sire, Rémy Mosseri

\section{To cite this version:}

Clément Sire, Rémy Mosseri. Spectrum of 1D quasicrystals near the periodic chain. Journal de Physique, 1989, 50 (24), pp.3447-3461. 10.1051/jphys:0198900500240344700 . jpa-00211154

\section{HAL Id: jpa-00211154 https://hal.science/jpa-00211154}

Submitted on 1 Jan 1989

HAL is a multi-disciplinary open access archive for the deposit and dissemination of scientific research documents, whether they are published or not. The documents may come from teaching and research institutions in France or abroad, or from public or private research centers.
L'archive ouverte pluridisciplinaire HAL, est destinée au dépôt et à la diffusion de documents scientifiques de niveau recherche, publiés ou non, émanant des établissements d'enseignement et de recherche français ou étrangers, des laboratoires publics ou privés. 
Classification

Physics Abstracts

$71.20+\mathrm{c}-71.25+\mathrm{e}-71.30+\mathrm{h}$

\title{
Spectrum of 1D quasicrystals near the periodic chain
}

\author{
Clément Sire and Rémy Mosseri \\ Laboratoire de Physique des Solides de Bellevue, CNRS, 92195 Meudon Cedex, France
}

(Reçu le 29 juin 1989, révisé le 4 septembre 1989, accepté le 6 septembre 1989)

\begin{abstract}
Résumé. - Nous étudions en perturbation un modèle de liaison forte sur une chaîne quasipériodique, au voisinage de la chaîne périodique, et cela au moyen d'une nouvelle numérotation des sites. Les principaux gaps sont bien décrits, alors que les très petits gaps ne sont bien rendus que pour une faible perturbation. Nous calculons exactement les énergies de la chaîne linéaire où naissent les gaps, et exhibons une numérotation naturelle qui les ordonne selon l'ordre décroissant. De plus, ce traitement permet de calculer de façon approchée la densité d'état électronique intégrée. Enfin, et en application de ces résultats, nous calculons au premier ordre, l'exposant $\delta$ qui décrit la façon dont la mesure du spectre tend vers zéro lorsque la taille de l'approximant tend vers l'infini. Au premier ordre, cet exposant ne dépend pas du quasicristal considéré.
\end{abstract}

\begin{abstract}
A tight binding model on the general 1D quasiperiodic chain is studied in the framework of perturbation theory, near the corresponding periodic chain, using a new set of coordinates. The main gaps are well described, whereas the very small ones are correctly given, only for a very small perturbation. For a given irrational number, the energies where the gaps appear in the periodic chain spectrum, are exactly derived. Moreover, a labelling for these gaps which orders them according to their decreasing width is naturally introduced, and an approached integrated density of states is explicitely written. As an application of this perturbative derivation, we give the first order expansion of $\delta$ the exponant which describes the vanishing of the total band width $B$ of an approximant, when its size increases : $B \sim 1 / n^{\delta}$. The first order expression for $\delta$ does not depend on the considered quasiperiodic chain.
\end{abstract}

\section{Introduction.}

The properties of the Schrödinger operator with a quasiperiodic potential is of considerable interest, since the discovery of quasicrystals. The electronic properties of quasiperiodic tilings in two or three dimensions are not yet well understood. In $1 \mathrm{D}$, which has been the most intensively studied case $[1,5,9]$, the spectrum in the framework of a tight binding model shows up interesting properties : it has an infinity of gaps and a zero Lebesgue measure. Moreover, the wave functions, instead of being extended or localized by the disorder, are lying on an intermediate state, that is, critical. Recently, Levitov [2] has studied the general 1D chain with two hopping constants 1 and $\rho$ distributed quasiperiodically. He works near $\rho=0$ and $\rho=+\infty$, that is when the energy levels are very close to the molecular states. 
Indeed, in both cases, the linear chain splits into an assembly of disconnected molecules. In this paper, we propose a general perturbative treatment near $\rho=1$, which gives the locus of the gaps, and describes the main gaps correctly.

\section{A new numbering on $1 D$ quasicrystals.}

Recently, a new set of coordinates has been introduced for vertices of approximants of 1D quasicrystals [3]. Consider an irrational number $\alpha$, and $\left(p_{\ell}, q_{\ell}\right)$ a sequence of approximants of $\alpha$

$$
\alpha_{\ell}=\frac{p_{\ell}}{q_{\ell}} \quad \text { and } \quad \lim _{\ell \rightarrow+\infty} \alpha_{\ell}=\alpha
$$

In the following we use the notation $n_{\ell}=p_{\ell}+q_{\ell}$. We call $S_{\infty}$ the linear chain obtained by associating a 1 to an horizontal bond and a $\rho$ to a vertical one, for each bond of the broken line drawn along the edges of a $\mathbb{Z}^{2}$ lattice into the band

$$
0 \leqslant y-\alpha x<1+\alpha \quad(x, y) \in \mathbb{Z}^{2} .
$$

This is exactly the well known cut and project method [4]. We take $0<\alpha<1$ without any loss of generality since, $S_{\infty}(1 / \alpha)$ is obtained from $S_{\infty}(\alpha)$ by inverting vertical and horizontal bonds. We define $S_{\ell}$ in the same way by replacing $\alpha$ by $\alpha_{\ell}$ in (2). We notice that $S_{\ell}$ is a periodic chain whose elementary cell contains $p_{\ell}+q_{\ell}$ atoms. The implicit assumption is that the properties of $S_{\ell}$ for $\ell \rightarrow+\infty$ will lead to the $S_{\infty}$ properties. It can be shown that the coordinates of points of $S_{\ell}$, in the band (before mapping), can be written as follows

$$
\begin{gathered}
\mathbf{X}_{n, m}=n \mathbf{a}_{\ell}+m \mathbf{b}_{\ell} \quad \text { with } \quad m \in \mathbb{Z}, \quad n=0,1, \ldots, n_{\ell-1} \\
\mathbf{a}_{\ell}=\left(\begin{array}{l}
q_{\ell}^{\prime} \\
p_{\ell}^{\prime}
\end{array}\right) \quad \mathbf{b}_{\ell}=\left(\begin{array}{l}
q_{\ell} \\
p_{\ell}
\end{array}\right)
\end{gathered}
$$

where $\left(\mathbf{a}_{\ell}, \mathbf{b}_{\ell}\right)$ is a unit basis of $\mathbb{Z}^{2}$ so that

$$
p_{\ell}^{\prime} q_{\ell}-p_{\ell} q_{\ell}^{\prime}=1
$$

$\mathbf{b}_{\ell}$ defines the unit cell of the approximant periodic structure and $\mathbf{a}_{\ell}$ is called a generator. In a subsequence of $p_{\ell}+q_{\ell}$ sites in $S_{\ell}$, we find one and only one $\mathbf{X}_{n, m}$, labelled with a fixed $n$. We give the example of the Fibonacci chain in figure 1. Now we look for the nearest neighbors of $\mathbf{X}_{n, m}$, in this new numbering :

$$
p_{\ell}^{\prime} \mathbf{b}_{\ell}-p_{\ell} \mathbf{a}_{\ell}=\left(\begin{array}{l}
1 \\
0
\end{array}\right) \text { and } q_{\ell} \mathbf{a}_{\ell}-q_{\ell}^{\prime} \mathbf{b}_{\ell}=\left(\begin{array}{l}
0 \\
1
\end{array}\right) .
$$

Thus, the two nearest neighbors of $\mathbf{X}_{n, m}$ are the two in the set

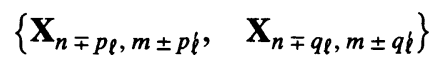

for which the first coordinate is between 0 and $n_{\ell}-1$. This two coordinates are $n+p_{\ell}\left[n_{\ell}\right]$ and $n+q_{\ell}\left[n_{\ell}\right]$ (where [ ] denotes the modulo operation). Let I be $\left[0, n_{\ell}-1\right]$. This last result can be rewritten in the following way.

* if $n-p_{\ell} \in \mathrm{I}$ and $n+p_{\ell} \in \mathrm{I}$, the site $n$ is surrounded by two 1 .

* if $n \pm p_{\ell} \in \mathrm{I}$ and $n \pm q_{\ell} \in \mathrm{I}$, the site $n$ is surrounded by a 1 and a $\rho$. 


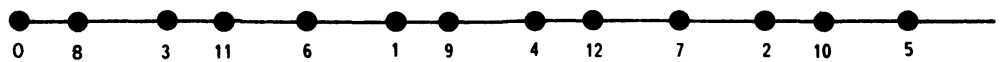

(b)

$\left(\begin{array}{ccccccccccccc}\lambda & \rho & 0 & 0 & 0 & 0 & 0 & 0 & 0 & 0 & 0 & 0 & \bar{\varepsilon} \\ \rho & \lambda & 1 & 0 & 0 & 0 & 0 & 0 & 0 & 0 & 0 & 0 & 0 \\ 0 & 1 & \lambda & \rho & 0 & 0 & 0 & 0 & 0 & 0 & 0 & 0 & 0 \\ 0 & 0 & \rho & \lambda & 1 & 0 & 0 & 0 & 0 & 0 & 0 & 0 & 0 \\ 0 & 0 & 0 & 1 & -\lambda & 1 & 0 & 0 & 0 & 0 & 0 & 0 & 0 \\ 0 & 0 & 0 & 0 & 1 & \lambda & \rho & 0 & 0 & 0 & 0 & 0 & 0 \\ 0 & 0 & 0 & 0 & 0 & \rho & \lambda & 1 & 0 & 0 & 0 & 0 & 0 \\ 0 & 0 & 0 & 0 & 0 & 0 & 1 & \lambda & \rho & 0 & 0 & 0 & 0 \\ 0 & 0 & 0 & 0 & 0 & 0 & 0 & \rho & \lambda & 1 & 0 & 0 & 0 \\ 0 & 0 & 0 & 0 & 0 & 0 & 0 & 0 & 1 & -\lambda & 1 & 0 & 0 \\ 0 & 0 & 0 & 0 & 0 & 0 & 0 & 0 & 0 & 1 & \lambda & \rho & 0 \\ 0 & 0 & 0 & 0 & 0 & 0 & 0 & 0 & 0 & 0 & \rho & \lambda & 1 \\ \varepsilon & 0 & 0 & 0 & 0 & 0 & 0 & 0 & 0 & 0 & 0 & 1 & -\lambda\end{array}\right)$

(c) $\left(\begin{array}{ccccccccccccc}\lambda & 0 & 0 & 0 & 0 & \bar{\varepsilon} & 0 & 0 & \rho & 0 & 0 & 0 & 0 \\ 0 & \lambda & 0 & 0 & 0 & 0 & 1 & 0 & 0 & \rho & 0 & 0 & 0 \\ 0 & 0 & \lambda & 0 & 0 & 0 & 0 & 1 & 0 & 0 & \rho & 0 & 0 \\ 0 & 0 & 0 & \lambda & 0 & 0 & 0 & 0 & 1 & 0 & 0 & \rho & 0 \\ 0 & 0 & 0 & 0 & \lambda & 0 & 0 & 0 & 0 & 1 & 0 & 0 & \rho \\ \varepsilon & 0 & 0 & 0 & 0 & -\lambda & 0 & 0 & 0 & 0 & 1 & 0 & 0 \\ 0 & 1 & 0 & 0 & 0 & 0 & -\lambda & 0 & 0 & 0 & 0 & 1 & 0 \\ 0 & 0 & 1 & 0 & 0 & 0 & 0 & -\lambda & 0 & 0 & 0 & 0 & 1 \\ \rho & 0 & 0 & 1 & 0 & 0 & 0 & 0 & \lambda & 0 & 0 & 0 & 0 \\ 0 & \rho & 0 & 0 & 1 & 0 & 0 & 0 & 0 & \lambda & 0 & 0 & 0 \\ 0 & 0 & \rho & 0 & 0 & 1 & 0 & 0 & 0 & 0 & \lambda & 0 & 0 \\ 0 & 0 & 0 & \rho & 0 & 0 & 1 & 0 & 0 & 0 & 0 & \lambda & 0 \\ 0 & 0 & 0 & 0 & \rho & 0 & 0 & 1 & 0 & 0 & 0 & 0 & \lambda\end{array}\right)$

Fig. 1. - (a) A Fibonacci chain of 13 sites, in the new numbering. (b) The Hamiltonian for a Fibonacci chain of 13 sites, in the classic indexation (left), and with the new numbering (right), as introduced in section 2 . We use the notation $\varepsilon=\mathrm{e}^{i k}$.

The case $n-q_{\ell} \in \mathrm{I}$ and $n+q_{\ell} \in \mathrm{I}$ is impossible since we took $\alpha<1$ and choose $\alpha_{\ell}<1$. Thus, this numbering orders the sites according to their local environment which is the relevant ordering in that case. We show in the next section that these new coordinates will be very useful to study a tight binding Hamiltonian on $S_{\ell}$.

\section{The Hamiltonian.}

We define the tight binding model on $S_{\infty}$ by the eigen equation

$$
t_{i+1} \Psi_{i+1}+x_{i} \Psi_{i}+t_{i} \Psi_{i-1}=E \Psi_{i}
$$

where $t_{i} \in\{1, \rho\}$ following $S_{\infty}$, and $x_{i}=\lambda$ if the site $i$ is surrounded by a $\rho$ and a 1 , $-\lambda$ otherwise. We show in figure 1 a typical Hamiltonian matrix on $S_{\ell}$ with the standard coordinates. Now, we use the result of section 2 to write the Hamiltonian in the new coordinates. We first introduce a Bloch 1D-vector $k$ since our system has period $n_{\ell}$. We have shown that the nearest neighbors of the site $i$ are the two sites among $i \pm p_{\ell}, i \pm q_{\ell}$, whose label lies between 1 and $\eta_{\ell}$. After some elementary manipulations, we obtain the following form for $\mathcal{H}_{\ell}$, the Hamiltonian matrix on $S_{\ell}$ :

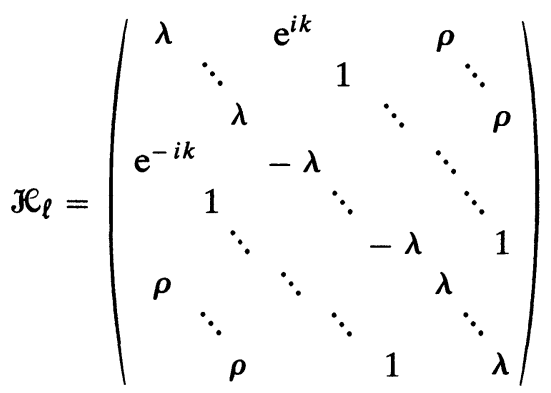

which is a multidiagonal matrix. If we define $h_{i, j}$ as the matrix elements of $\mathcal{H}_{\ell}$, this reads

$$
\begin{aligned}
h_{i, i} & =\lambda \quad \text { if } 1 \leqslant i \leqslant p_{\ell} \text { or } q_{\ell}<i \leqslant p_{\ell}+q_{\ell}=n_{\ell} \\
& =-\lambda \text { otherwise } \\
h_{i, i \pm p_{\ell}} & =1 \text { when } i \pm p_{\ell} \in\left[2, n_{\ell}\right] \\
h_{p_{\ell}+1,1} & =\bar{h}_{1, p_{\ell}+1}=\mathrm{e}^{-i k} \\
h_{i, i \pm q_{\ell}} & =\rho \text { when } i \pm q_{\ell} \in\left[1, n_{\ell}\right]
\end{aligned}
$$


$\mathscr{H}_{\ell}$ is much more symmetric than the equivalent matrix in the classical numbering, one can call the geometric ordering (Fig. 1). For the calculation, we have applied the Bloch theorem after noticing that $\mathbf{X}_{0, m}$, which is the first atom of the considered cell interacts with $\mathbf{X}_{q \ell, m-q \ell}$ and $\mathbf{X}_{p_{\ell}, m-p_{\ell}}$, which is the last atom of the preceding cell. Thus, $k$ is the Bloch vector which lies in the range $k \in[0, \pi]$. Since $\mathcal{H}_{\ell}$ has a priori, $n_{\ell}$ eigenvalues for a fixed $k$, the spectrum of $S_{\ell}$ is of the form

$$
S p\left\{S_{\ell}\right\}=\left\{E_{j}(\cos k), k \in[0, \pi], j=1, \ldots, n_{\ell}\right\}
$$

and consists in $n_{\ell}$ bands which may overlap (Fig. 3). The bands edges are given by $E_{j}^{ \pm}$which are in the set $\left\{E_{j}( \pm 1), j \in\left[1, n_{\ell}\right]\right\}$, that is, for $k=0$ and $k=\pi$ (in general, we do not have $E_{j}^{ \pm}=E_{j}( \pm 1)$, with the same label $\left.j\right)$. Indeed, the secular equation $\operatorname{det}\left(\mathscr{H}_{\ell}-E J\right)=0$, can be written $P_{\ell}(E)=\cos k$. So, the bands are given by the condition $\left|P_{\ell}(E)\right| \leqslant 1$ and their edges by $\left|P_{\ell}(E)\right|=1$. In order to apply the perturbation theory to $\mathcal{H}_{\ell}$, we rewrite the Hamiltonian as

$$
\mathscr{H}_{\ell}=\mathscr{H}_{\ell}^{(0)}+(\rho-1) \Delta_{\ell}+\lambda \Delta_{\ell}^{\prime}
$$

where $\mathfrak{H}_{\ell}^{(0)}$ is the Hamiltonian at $\lambda=0, \rho=1 . \Delta_{\ell}$ and $\Delta_{\ell}^{\prime}$ are the matrices defined by

$$
\begin{array}{rll}
\Delta_{\ell}(i, j)=1 & \text { if } & |i-j|=q_{l} \\
\Delta_{\ell}^{\prime}(i, i)=1 & \text { if } & 1 \leqslant i \leqslant p_{\ell} \text { or } q_{\ell}<i \leqslant n_{\ell} \\
-1 & \text { if } & p_{\ell}<i \leqslant q_{\ell}
\end{array}
$$

the other coefficients being equal to zero.

\section{The spectrum of a $1 D$ quasicrystal.}

In this section we are interested in the derivation of $E_{j}^{+}(\rho, \lambda)$ for $\lambda \neq 0$ and $\rho \neq 1$. We first give $E_{j}^{ \pm}(\rho, \lambda)$ to the first order in $(1-\rho)$ and $\lambda$, before studying the validity of the method. We first recall the classical result at $\lambda=0$ and $\rho=1$ :

* $k=0$ : we obtain the $n_{\ell}$-polygonal chain. $\mathfrak{H}_{\ell}$ is then a cyclic matrix whose eigenvalues are $E_{j}=2 \cos \left(2 j \pi p_{\ell} / n_{\ell}\right)$, which are degenerate of order 2 (except for $j=0$ and $j=n_{\ell} / 2$, if $n_{\ell}$ is even), whose eigenvectors at site $m \mathrm{read}:|j, \pm\rangle_{m}=\mathrm{e}^{ \pm 2 i \pi m j / n_{\ell}} / \sqrt{n_{\ell}}$.

$* k=\pi$ : the energy levels are given by $E_{j}^{\prime}=2 \cos \left(2 \pi\left(j p_{\ell}+1 / 2\right) / n_{\ell}\right)$ and have the opposite sign of the preceding ones for $n_{\ell}$ odd.

So, in the following, in order to have compact formulae, we take $n_{\ell}$ odd. If $\alpha$ is irrational, when $n_{\ell}$ goes to infinity, we expect the result for $n_{\ell}$ and $n_{\ell}+1$ to be the same. In the case $\alpha \in \mathbf{Q}$ with small $p$ and $q$, and $p+q$ even, we cannot make that assumption. Anyway, the calculations which follows can be done with $n_{\ell}$ even, by studying separately the cases $k=0$ and $k=\pi$. Before studying the perturbed chain, we show in figure 3 the band structure for the periodic linear chain, which is obtained by folding $n_{\ell}$ times the well known graph of the dispersion relation, namely $E(k)=2 \cos k$.

Now, we study the case $0<|\lambda| \ll 1$ and $|1-\rho| \ll 1$. We use the perturbation method for degenerated states. In the eigenspace associated to $E_{j}$, straightforward calculations give for $k=0$.

$$
\begin{array}{r}
\left\langle j, \varepsilon\left|\mathcal{H}_{\ell}\right| j, \varepsilon\right\rangle=2 \cos \left(\frac{2 \pi j p_{\ell}}{n_{\ell}}\right)\left(1-(1-\rho) \frac{p_{\ell}}{n_{\ell}}\right)+\lambda\left(\frac{4 p_{\ell}}{n_{\ell}}-1\right) \\
\left\langle j, \varepsilon\left|\mathcal{H}_{\ell}\right| j,-\varepsilon\right\rangle=\frac{2 e^{-\frac{2 \varepsilon i j \pi}{n_{\ell}}}}{n_{\ell} \sin \left(\frac{2 \pi j}{n_{\ell}}\right)}\left(\lambda \sin \left(\frac{4 \pi p_{\ell} j}{n_{\ell}}\right)-(1-\rho) \sin \left(\frac{2 \pi p_{\ell} j}{n_{\ell}}\right)\right)
\end{array}
$$


For $\varepsilon= \pm 1$. Now, for each energy (except for $E= \pm 2$ ), we have to diagonalize a $2 \times 2$ matrix. We find the following eigenenergies which gives the bands edges.

$$
\begin{aligned}
E_{j}^{(0) \pm} & =2 \cos \left(\frac{2 \pi j p_{\ell}}{n_{\ell}}\right)\left(1-(1-\rho) \frac{p_{\ell}}{n_{\ell}}\right)+\lambda\left(\frac{4 p_{\ell}}{n_{\ell}}-1\right) \\
& \pm \frac{2}{n_{\ell} \sin \left(\frac{2 \pi}{n_{\ell}}\right)}\left[(1-\rho) \sin \left(\frac{2 \pi j p_{\ell}}{n_{\ell}}\right)-\lambda \sin \left(\frac{4 \pi j p_{\ell}}{n_{\ell}}\right)\right] \\
E_{j}^{(\pi) \pm}= & -2 \cos \left(\frac{2 \pi j p_{\ell}}{n_{\ell}}\right)\left(1-(1-p) \frac{p_{\ell}}{n_{\ell}}\right)+\lambda\left(\frac{4 p_{\ell}}{n_{\ell}}-1\right) \\
& \pm \frac{2}{n_{\ell} \sin \left(\frac{2 \pi j}{n_{\ell}}\right)}\left[(1-\rho) \sin \left(\frac{2 \pi j p_{\ell}}{n_{\ell}}\right)+\lambda \sin \left(\frac{4 \pi j p_{\ell}}{n_{\ell}}\right)\right]
\end{aligned}
$$

while the spectrum edges are given by

$$
\begin{aligned}
& E^{(0)}=2\left(1-(1-\rho) \frac{p_{\ell}}{n_{\ell}}\right)+\lambda\left(\frac{4 p_{\ell}}{n_{\ell}}-1\right) \\
& E^{(\pi)}=-2\left(1-(1-\rho) \frac{p_{\ell}}{n_{\ell}}\right)+\lambda\left(\frac{4 p_{\ell}}{n_{\ell}}-1\right)
\end{aligned}
$$

which are given by the average of the potential. We want now to discuss the validity of our treatment. It is known that a perturbative approach is correct provided the typical spacing between non perturbed levels is large enough compared to the matrix elements of the perturbative potential. Here, this condition reads :

$$
|\lambda|+|1-\rho| \ll \frac{\pi^{2}}{n_{\ell}} .
$$

So, we expect that for an approximant $p_{\ell} / q_{\ell}$ of $\alpha$ with $p_{\ell}+q_{\ell} \leqslant \frac{\pi^{2}}{|\lambda|+|1-\rho|}$ the opening gaps will be correctly described by (10)-(10'). In particular, if $\alpha=p / q$ is a rational number, the calculation is correct provided $\lambda$ and $\rho$ verify (11) with $n_{\ell}=p+q$. Now we assume $\alpha$ being an irrational number. Then, to recover the exact quasicrystal, $\ell$ and $n_{\ell}$ must go to infinity, so that for fixed $\rho$ and $\lambda$, the condition (11) is never verified. So at first sight, our derivation cannot apply to the case $\alpha \neq \mathbf{Q}$. In fact, we shall see that for large $n_{\ell}$, the main gaps which have already appeared for small $n_{\ell}$, do not change any more. (The only change is that in formulae (10)- $\left(10^{\prime}\right) p_{\ell} / n_{\ell}$ must be replaced by $\left.\frac{\alpha}{1+\alpha}\right)$. So if $\alpha$ is an irrational number, if $p / q$ is a best approximant of $\alpha$, and if $|\lambda|+|1-\rho|<\pi^{2} /(p+q)$, then, the $p+q$ main gaps are given by formulae (10)-(10'). From now, we differenciate $\ell$ used in formulae (10)-(10') and $\ell^{\prime}$, which is the index of the linear chain under study. Our result is that when $\ell^{\prime}$ goes to infinity, the properties of the spectrum are correctly given by $(10)-\left(10^{\prime}\right)$, with $\ell$ a finite number (at least the $n_{\ell}$ first gaps). Moreover, since main gaps are already obtained for small approximants, for which the corresponding linear chain have quite spaced energy levels, we expect them to be well described by our treatment even for $|1-\rho|+|\lambda|$ quite large. To illustrate our statement, we consider the Fibonacci chain for which a trace mapping can be found, which allows us to 
find numerically the spectrum of $S_{\ell}$ very easily. One can show that an energy $E$ is in the spectrum of $S_{\ell}$ (we call it $S p\left\{S_{\ell}\right\}$ ), if half the modulus of the trace of the transfert matrices product is less than 1 . It reads explicitely :

$$
\begin{array}{rlrl} 
& \text { with } & x_{0} & =\frac{1}{2 \rho}(E-3 \lambda) \\
E \in S p\left\{S_{\ell}\right\} \leftrightarrow\left|x_{\ell}(E)\right| \leqslant 1 & x_{1} & =\frac{1}{2}(E+\lambda) \\
x_{2} & =\frac{1}{2 \rho}\left((E-\lambda)^{2}-1-\rho^{2}\right) \\
\text { and } \quad x_{\ell+3} & =2 x_{\ell+2} x_{\ell+1}-x_{\ell} .
\end{array}
$$

For example, we found (Fig. 2) that for $n_{\ell}=55$, the 55 main gaps are very correctly given for $|\lambda|+|1-\rho| \leqslant 0.2$, while for $0.2 \leqslant|\lambda|+|1-\rho|<0.3$ many gaps remain good although some of them (especially the little gaps surrounding the biggest ones) are very badly recovered. If we only keep the ten main gaps, they are correctly described for $|\lambda|+|1-\rho| \leqslant 0.4$ (Fig. 2), where the linear approximation cannot be good any longer. In order to be complete, we give the equivalent result for the model which was originally introduced by Kohmoto et al. and Ostlund et al. [5]. In this case, the chain $S_{\ell}$ is built by associating a diagonal hopping constant $\lambda$ to each horizontal bond, and a diagonal hopping constant $-\lambda$ to each vertical one, in the band we have considered in section 2 , in the cut and project framework. The result we obtain is the following :

$$
\begin{gathered}
E_{j}^{(0) \pm}=2 \cos \left(\frac{2 \pi j p_{\ell}}{n_{\ell}}\right)+\lambda\left(\frac{2 p_{\ell}}{n_{\ell}}-1\right) \pm \frac{2 \lambda \sin \left(\frac{2 \pi j p_{\ell}}{n_{\ell}}\right)}{n_{\ell} \sin \left(\frac{2 \pi j}{n_{\ell}}\right)} \\
E_{j}^{(\pi) \pm}=-2 \cos \left(\frac{2 \pi j p_{\ell}}{n_{\ell}}\right)+\lambda\left(\frac{2 p_{\ell}}{n_{\ell}}-1\right) \pm \frac{2 \lambda \sin \left(\frac{2 \pi j p_{\ell}}{n_{\ell}}\right)}{n_{\ell} \sin \left(\frac{2 \pi j}{n_{\ell}}\right)}
\end{gathered}
$$

while the spectrum edges are

$$
\begin{aligned}
& E^{(0)}=2+\lambda\left(\frac{2 p_{\ell}}{n_{\ell}}-1\right) \\
& E^{(\pi)}=-2+\lambda\left(\frac{2 p_{\ell}}{n_{\ell}}-1\right) .
\end{aligned}
$$

For $\alpha$ being the golden mean, the spectrum of the associate Fibonacci chain is given by

$$
\begin{array}{rlrl} 
& \text { with } \quad x_{-1}(E) & =1 \\
x_{0}(E) & =\frac{1}{2}(E-\lambda) \\
E \in S p\left\{S_{\ell}\right\} \leftrightarrow\left|x_{\ell}(E)\right| \leqslant 1 & x_{1}(E) & =\frac{1}{2}(E+\lambda) \\
\text { and } & x_{\ell+3} & =2 x_{\ell+2} x_{\ell+1}-x_{\ell} .
\end{array}
$$

The result is shown in figure 2 and the conclusions are the same as in the preceding case. Now, 
$N=13$ Gaps: 13
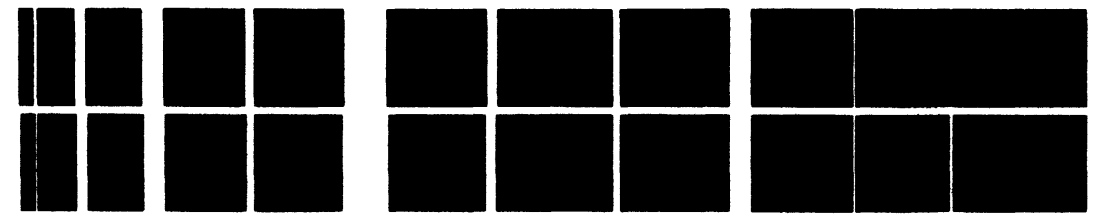

$M=21 \quad$ Gaps: 21
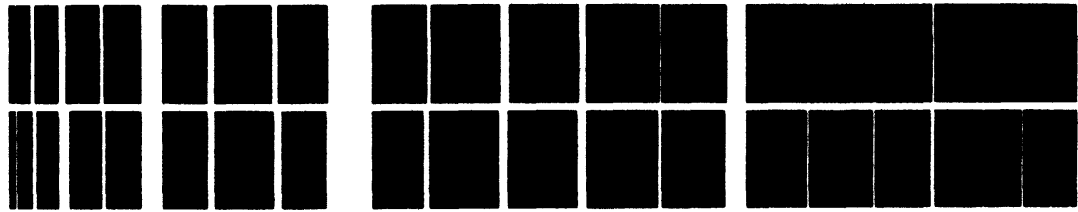

$N=55 \quad$ Gaps: 55

a)
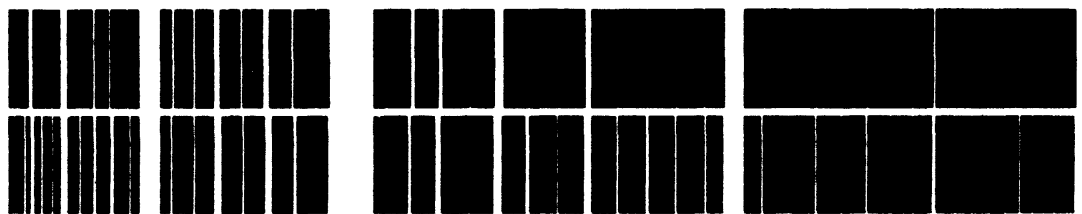

$M=13 \quad$ Gaps: 13
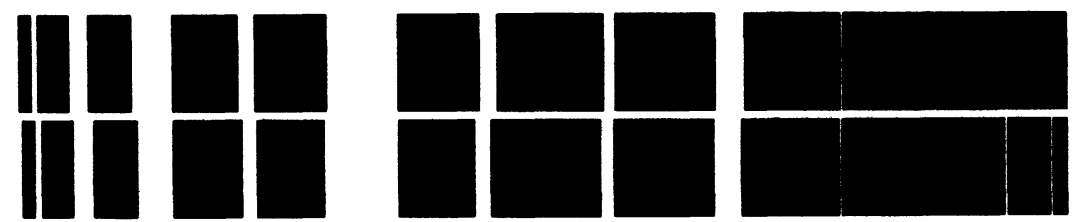

$M=21 \quad$ Gaps: 21
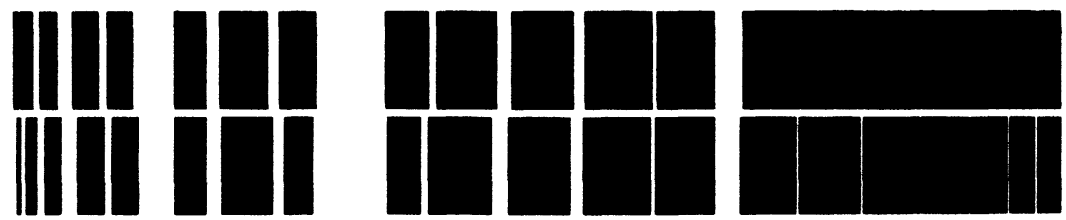

$M=55 \quad$ Gaps: 55

b)
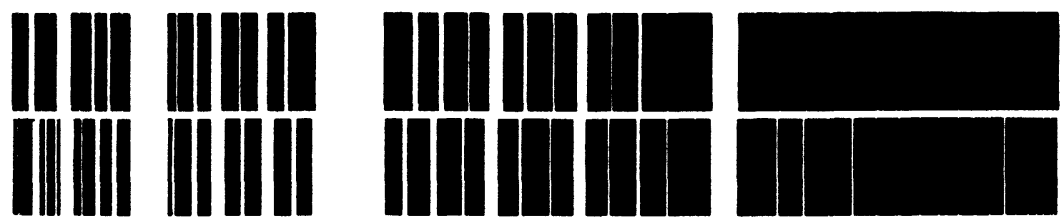

Fig. 2. - The numerical spectra of a Fibonacci chain for $N=13,21,55$ sites (upper spectra) are compared to our results (10) $\left(10^{\prime}\right)$ for (a) $\rho=0.9, \lambda=0.05 ;$ (b) $\rho=0.85, \lambda=0.1$; (c) $\rho=0.7, \lambda=0.1$. (d) the spectrum of the Fibonacci chain defined by (14) compared to (13) (13'), for $\lambda=0.15$. 
$N=13 \quad$ Gaps: 13
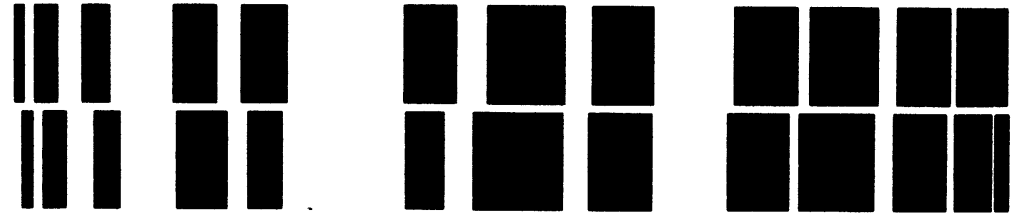

$M=21 \quad$ Gaps: 13
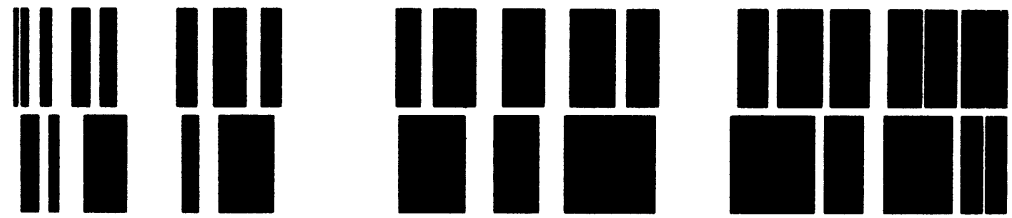

$N=55 \quad$ Gaps: 13

c)
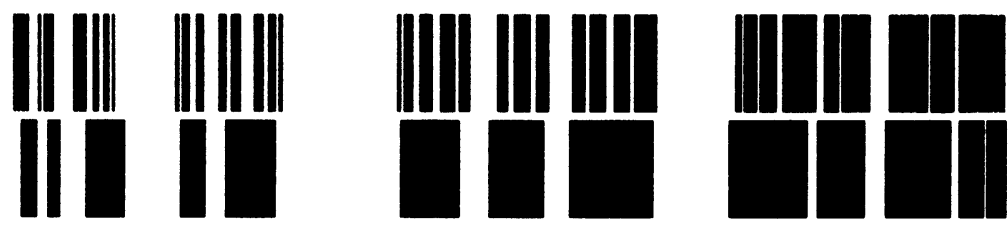

$H=55$ Gaps: 55

d)
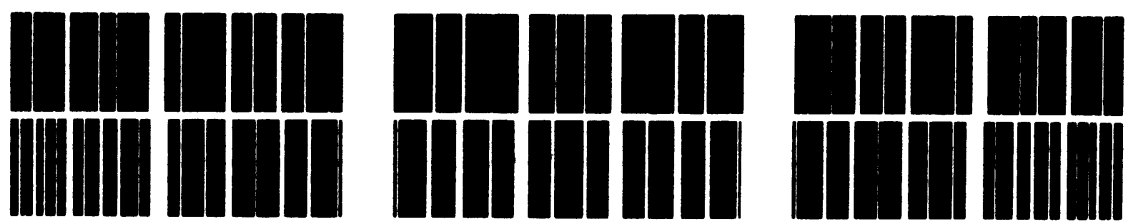

Fig. 2 (continued)

since we have shown that with certain conditions, our treatment is in very good agreement with numerical datas, we study some important applications of our results.

\section{Indexation of gaps.}

Consider an energy of the periodic linear chain labelled by $j$ i.e. $E_{j}^{(\ell)}=2 \cos \left(\frac{2 \pi j p_{\ell}}{n_{\ell}}\right)$. We can see that the $E_{j}^{(\ell)}$ s are not labelled following the increasing order along the energy axis, but in such a way that for a given $j$ we have

$$
\left|E_{j}^{(\ell+1)}-E_{j}^{(\ell)}\right| \sim \frac{4 \pi j}{n_{\ell} n_{\ell+1}}\left|\sin \left(\frac{2 \pi j p_{\ell+1}}{n_{\ell+1}}\right)\right|
$$

when $\ell \rightarrow+\infty$ 
where we have assumed that $\frac{p_{\ell}}{q_{\ell}}$ is a best approximant of $\alpha$, so that we have $\left|p_{\ell+1} q_{\ell}-p_{\ell} q_{\ell+1}\right|=1$. This difference has to be compared to the distance between $E_{j}^{(\ell+1)}$ and its nearest neighbouring energies $2 \cos \left(\frac{2 \pi\left(j p_{\ell+1} \pm 1\right)}{n_{\ell+1}}\right)$. A simple calculation gives

$$
\begin{aligned}
& \left|E_{j}^{(\ell+1)}-2 \cos \left(\frac{2 \pi\left(j p_{\ell+1} \pm 1\right)}{n_{\ell+1}}\right)\right| \sim \frac{4 \pi}{n_{\ell+1}}\left|\sin \left(\frac{2 \pi j p_{\ell+1}}{n_{\ell+1}}\right)\right| \\
& \text { when } \ell \rightarrow+\infty
\end{aligned}
$$

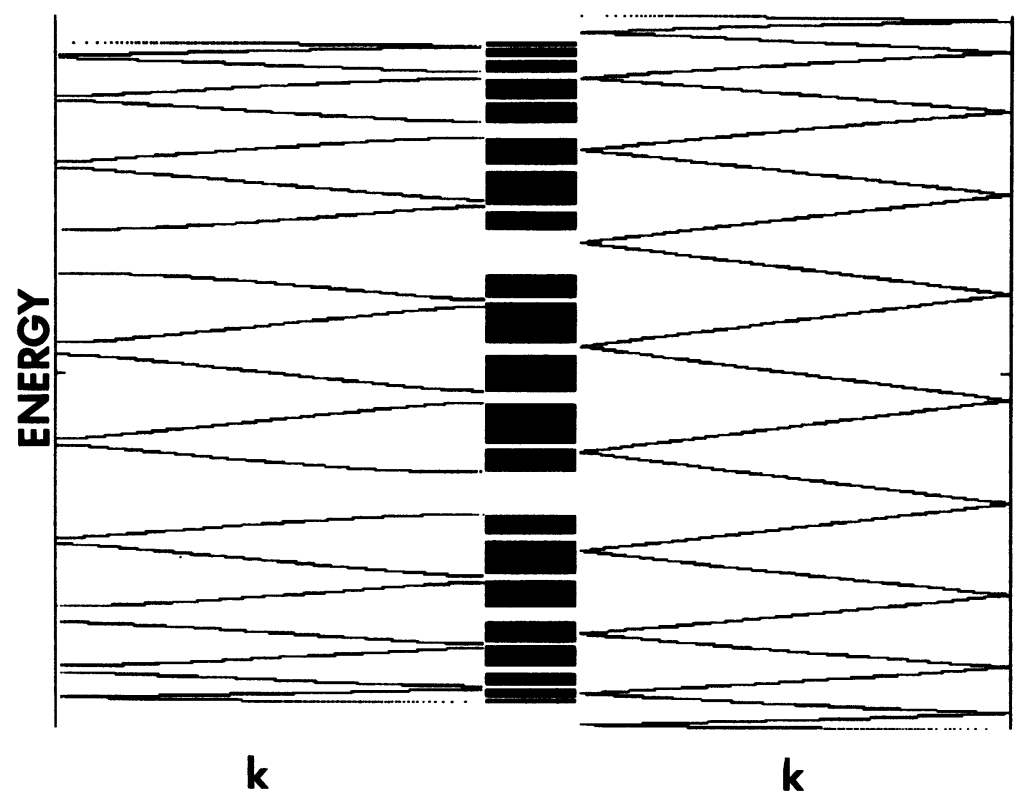

Fig. 3. - The band structure for a linear chain of 21 sites (right) and for a Fibonacci chain (left) with $\rho=0.8, \lambda=0$, and 21 sites is shown. We have also shown the gaps given by (10) $\left(10^{\prime}\right) . k$ is the Bloch vector.

Comparing (15) and (16), and since $1 \leqslant j \leqslant\left|\left(n_{\ell}-1\right) / 2\right|$, we see that $E_{j}^{(\ell+1)}$ can be naturally associated to $E_{j}^{(\ell)}$, and will correspond to the same opening gap. So, for a fixed $j$, we see that in the spectrum of the periodic chain $(E \in[-2,2])$, a gap will open for the infinite quasiperiodic chain at the energies

$$
G_{2 j}^{ \pm}= \pm \lim _{\ell \rightarrow+\infty} E_{j}^{(\ell)}= \pm 2 \cos \left(2 \pi j \frac{\alpha}{1+\alpha}\right) \quad j \geqslant 1
$$

We can make the same treatment for $j^{\prime}=\left[\frac{n_{\ell}+1}{2}\right]-j$ (where [ ] denotes the integer part), and we obtain gaps at

$$
G_{2 j-1}^{ \pm}= \pm 2 \cos \left((2 j-1) \pi \frac{\alpha}{1+\alpha}\right) \quad j \geqslant 1
$$


Moreover, the opening of the gaps for given $\rho, \lambda$, and $j$ are given by (10)-(10') for $\ell \rightarrow+\infty$. Of course, the gap opening at $G_{j}^{ \pm}$will be correctly given for

$$
j \leqslant \frac{\pi^{2}}{|\lambda|+|1-\rho|} .
$$

We write explicitely the formula we obtain when $j$ obeys this last condition. The gap edges are given by

$$
\begin{aligned}
& 2 \varepsilon \cos (\pi j \theta)(1-(1-\rho) \theta)+\lambda(4 \theta-1) \\
& +\frac{2 \varepsilon^{\prime}}{\pi j}[(1-\rho) \sin (\pi j \theta)-\varepsilon \lambda \sin (2 \pi j \theta)]
\end{aligned}
$$

and the spectrum edges are

$$
2 \varepsilon(1-(1-\rho) \theta)+\lambda(4 \theta-1)
$$

where we define $\theta=\frac{\alpha}{(1-\alpha)}$ and $\varepsilon, \varepsilon^{\prime} \in\{-1,1\}$. Finally, we see that the gaps are indexed by only one integer $j$ which is the "memory » of the periodic linear chain. They open at $G_{j}^{+}$as soon as $\lambda \neq 0$ or $\rho \neq 1$. This indexation is very interesting since it labels the gaps according to their intensity if we do not mind the sine term. In figure 4, we show the main gaps and their labels, for a Fibonacci chain with $\lambda=0$ and $\rho=0.9$. Now, we focus on the density of states (DOS) or more precisely, the integrated DOS. Between two gaps labelled by $j$ and $j^{\prime}$, there are exactly the same number of states, whatever $\rho$ and $\lambda$ are, since it can be shown [6] that the bands never overlap. Thus, in the integrated DOS, the height of the plateau corresponding to the $j$-th gap does not depend on $\rho$ and $\lambda$. Let us define the DOS $n(E)$, and $g(E)$, the integrated DOS, normalized in such a way that we have

$$
g(E)=\int_{-\infty}^{E} n(E) \mathrm{d} E \text { and } \lim _{E \rightarrow+\infty} g(E)=1 .
$$

Then, we call $G_{j}^{ \pm}(\rho, \lambda)$ the center of the $j$-th gap. With our definitions, we have naturally $G_{j}^{ \pm}(1,0)=G_{j}^{ \pm}$, which has been previously defined. Thus, for any given $\rho$ and $\lambda$, we can write

$$
\begin{aligned}
& g\left(\max \left(G_{j}^{+}(\rho, \lambda), G_{j}^{-}(\rho, \lambda)\right)\right)=\max (-j \theta[1], j \theta[1]) \\
& g\left(\min \left(G_{j}^{+}(\rho, \lambda), G_{j}^{-}(\rho, \lambda)\right)\right)=\min (-j \theta[1], j \theta[1])
\end{aligned}
$$

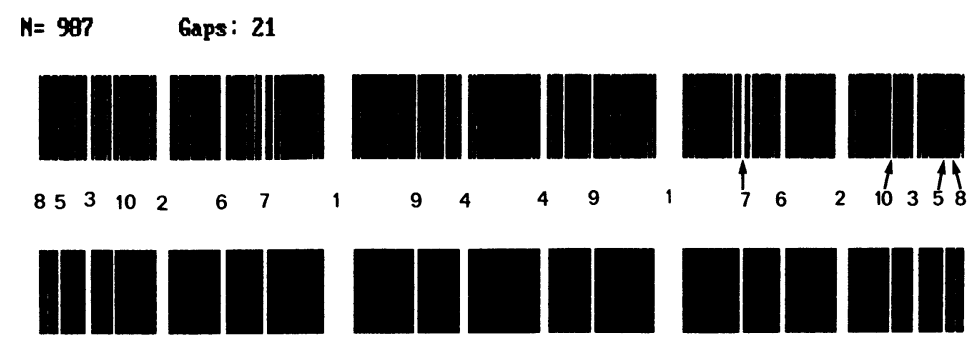

Fig. 4. - The labelling of the main gaps for a Fibonacci chain of 987 atoms, compared to the 21 main gaps predicted by (17) $\left(17^{\prime}\right)$, for $\rho=0.9, \lambda=0$. The fifth gap is quite small because of the sine term in (17). 
Pilonecel $r=.65 \quad 610$ atoms.

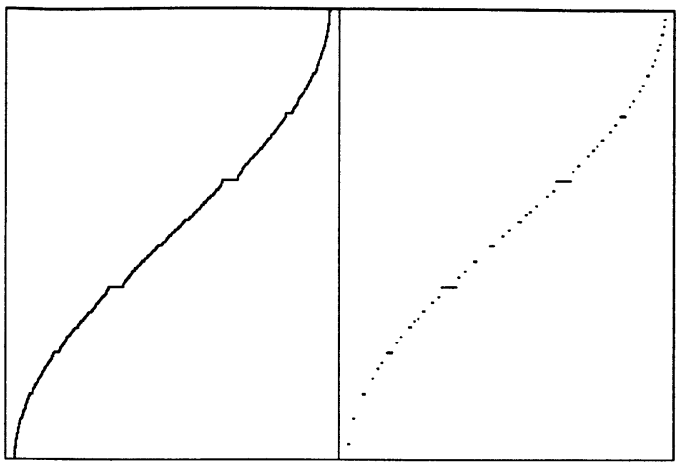

a)
Octonecel $s=.85 \quad 5 n$ atoms.

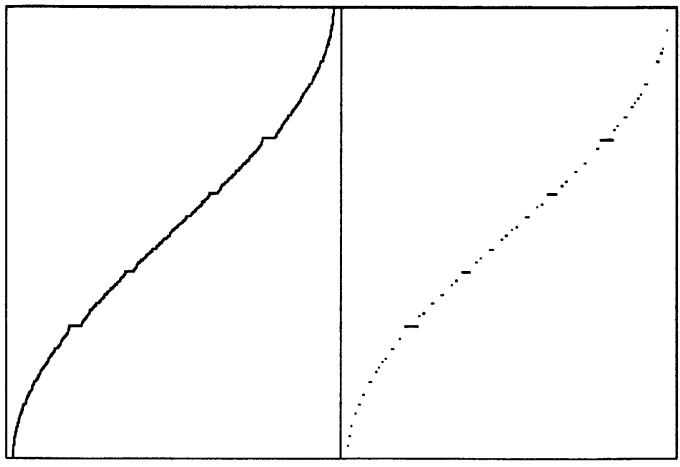

b)

Fig. 5. - (a) The IDOS of a large Fibonacci chain (left) and the IDOS given by (17) $\left(17^{\prime}\right)\left(17^{\prime \prime}\right)$ for $\rho=0.85, \lambda=0$. (b) The IDOS of a large Octonacci chain (left) and the IDOS given by (17) $\left(17^{\prime}\right)\left(17^{\prime \prime}\right)$ for $\rho=0.85, \lambda=0$.

In figure 5, we show the integrated DOS for two different quasiperiodic chain related respectively, to $\alpha=\frac{\sqrt{5}-1}{2}$ and $\alpha=\sqrt{2}-1$. The agreement between the numerically

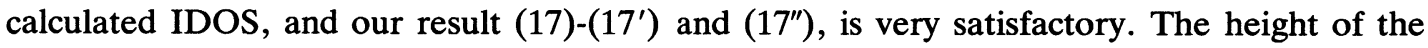
plateaux are exactly given for any value of $\rho$ (we took $\lambda=0$ ), while their width is correct only for the first gaps, whose label $j$ verifies (11). We note that Belissard, and Kalugin et al. [7], found similar results for the height of the plateaux, invoking more complicated tools.

We want now to specify the conditions for which this formula apply when $\alpha \in \mathbf{Q}$, that is for finite approximant. If we come back to equations (10)-(10') and (13) for $\alpha=p / q$, we see that in formulae (17)-(17') we only have to take $1 \leqslant j \leqslant(p+q)-1=n-1$. Even if formulae (17)-(17') only hold for $p$ and $q$ infinite, we show that even for small $p$ and $q$ they are quite correct. The main discrepancy between both formulae is for $j=1$ (we recall that formulae (10), (11), (13) are correct for $n$ odd, whereas (17) and (17') hold for any $n$ ). Then, for $j=1$ we find a relative discrepancy for the bands edges of the order $\pi / 6 n$. Thus, already for quite small $n$ our result (17)-(17') is correct. The width of the gap is badly approximated for $n=2(4|1-\rho| / \pi$ instead of $2|1-\rho|)$, better given for $n=3$ and good for $n \geqslant 5$. However, if we want to describe the gaps for very small values of $n$, the formulae (10)-(11) give the good result. (17)-(17') must be seen as simplified formulae which hold for large $n$, and especially for $n$ infinite.

We now study a second particular case, namely $\theta \ll 1$, which corresponds to very small (or large) $\alpha$. Then, we obtain a linear chain with one defect for about Int $\left[\frac{1}{\alpha}\right]$ (or Int $[\alpha]$ ) atoms (where Int $[x]$ is the integer part of $x$ ). Equation (17) shows that for $|1-\rho| \leqslant \theta$ (we take $\lambda=0$ ) the main gaps have approximately a constant width $\Delta \simeq 4 \theta(1-\rho)$ and are located at $\pm 2 \cos (\pi j \theta), j \ll 1 / \theta$. We numerically found the eigenvalues of $\mathscr{H}_{\ell}$ in (7), for $p_{\ell}=6 q_{\ell}=95$ and $\theta=6 / 101$, and show in table $\mathrm{I}$, the 5 main gaps for $\rho=0.9$. They are well described and verify the announced property.

\section{The measure of the spectrum.}

In the preceding section, we have shown that near $\rho=1$ and $\lambda=0$, the spectrum of a linear quasiperiodic chain has an infinity of gaps which appear at $G_{j}^{ \pm}= \pm 2 \cos (\pi j \theta)$. Since 
Table I. - The five first main gaps (center and width) for the chain defined in section 5. The subscript "n.s. " denotes numerical simulations, whereas "th. " refers to equations (17), $\left(17^{\prime}\right)$. The width is near $4 \theta(1-\rho) \sim 0.023762 \ldots$

$\begin{array}{ccccc}\text { Gap } & E_{\text {n.s. }} & \Delta E_{\text {n.s. }} & E_{\text {th. }} & \Delta E_{\text {th. }} \\ & & & & \\ 1 & 1.9543 & 0.0238 & 1.9536 & 0.0236 \\ 2 & 1.8513 & 0.0230 & 1.8512 & 0.0232 \\ 3 & 1.6843 & 0.0230 & 1.6846 & 0.0225 \\ 4 & 1.4590 & 0.0224 & 1.4594 & 0.0216 \\ 5 & 1.1832 & 0.0213 & 1.1835 & 0.0205\end{array}$

$\{j \pi \theta[2 \pi] j \geqslant 1\}$ is dense in [0,2 $\pi]$ for $\theta$ an irrational number, points where gaps open are also dense in $[-2,2]$. Thus, the question of evaluating the total width of the bands arises. Numerical studies were performed for the quasiperiodic linear chain obtained for $\alpha=\frac{\sqrt{5}-1}{2}$ [5] and $\alpha=\sqrt{2}-1$, in the framework of the study of a quasicrystal, related to the octagonal quasiperiodic tiling [8]. In both cases, the measure of the spectrum were found to be zero and $B_{\ell}$, the measure of the spectrum of $S_{\ell}$, found to vanish like $n_{\ell}^{-\delta_{\alpha}(\rho, \lambda)}$. Recently, it has been proved rigourously that the Lebesgue's measure is zero for any $\alpha$, in the case $\rho=1, \lambda \neq 0$ [10]. We show in the following that our study leads to an expression for $\delta_{\alpha}(\rho, \lambda)$, near $\rho=1$ and $\lambda=0$. For a crystal $\delta_{\alpha}(1,0)=0$, while for the corresponding quasicrystal $\delta_{\alpha}(\rho, \lambda)$ is a smooth function of $\rho$ and $\lambda$, depending a priori on $\alpha$, and which verifies

$$
\lim _{\lambda \rightarrow \pm \infty} \delta_{\alpha}\left(\rho_{0}, \lambda\right)=\lim _{\rho \rightarrow \pm \infty} \delta_{\alpha}\left(\rho, \lambda_{0}\right)=\lim _{\rho \rightarrow 0} \delta_{\alpha}\left(\rho, \lambda_{0}\right)=+\infty
$$

for any fixed $\lambda_{0}$ and $\rho_{0}$. Indeed, in these cases, the linear chain splits in an assembly of molecules, and its spectrum reduces to a finite number of molecular states. So, even for finite $\ell$, the spectrum measure is zero. From now, we study the case $\lambda=0$ and $\rho \neq 1$, and will give the result for $\lambda \neq 0$ and $\rho=1$, which can be derived in the same way. In the following, we take $\rho$ such as $|1-\rho| \leqslant 1 / n_{\ell}^{2}$, so that our calculation is expected to be quite correct. From (17)-(17'), the total band width is

$$
\frac{B_{\ell}}{B_{0}} \sim 1-|1-\rho|\left[\theta_{\ell}+2 \sum_{j=1}^{n_{\ell}} \frac{\left|\sin \left(\pi j \theta_{\ell}\right)\right|}{\pi j}\right]
$$

where

$$
\theta_{\ell}=\frac{\alpha_{\ell}}{1+\alpha_{\ell}}=\frac{p_{\ell}}{n_{\ell}}
$$

Using the Euler formula, the term between brackets can be written

$$
\frac{2}{\pi} \int_{0}^{p_{\ell}} \frac{|\sin (\pi x)|}{x} \mathrm{~d} x+h(\theta)+O\left(\frac{1}{n_{\ell}}\right)
$$

The function $h(\theta)$ is a series of the variable $\theta$, whose convergence radius is found to be 1 . 
Moreover, one can write

$$
\begin{aligned}
\int_{0}^{p_{\ell}} \frac{|\sin (\pi x)|}{x} \mathrm{~d} x & =f(\theta)+\int_{0}^{1}|\sin \pi x| \mathrm{d} x \times \ln p_{\ell}+O\left(\frac{1}{n_{\ell}}\right) \\
& =f(\theta)+\frac{2}{\pi} \ln p_{\ell}+O\left(\frac{1}{n_{\ell}}\right)
\end{aligned}
$$

We then obtain, grouping the constant terms in a generic function $F(\theta)$ and since $\ln p_{\ell} \sim \ln n_{\ell}$ for large $n_{\ell}$

$$
\frac{B_{\ell}}{B_{0}} \sim 1-\frac{4|1-\rho|}{\pi^{2}}\left(F(\theta)+\ln n_{\ell}\right)+o(|1-\rho|)
$$

Thus, for large $\ell$, and since we choose $\ln n_{\ell} \ll|1-\rho|^{-1}$, we can write

$$
\begin{aligned}
\ln \frac{B_{\ell}}{B_{0}} & \sim \ln \left(1-\frac{4|1-\rho|}{\pi^{2}} \ln n_{\ell}\right)+o(|1-\rho|) \\
& \sim-\frac{4|1-\rho|}{\pi^{2}} \ln n_{\ell}+o(|1-\rho|)
\end{aligned}
$$

which gives the first order expansion of $\delta_{\alpha}(\rho, 0)$ in power of $|1-\rho|$

$$
\begin{aligned}
\delta_{\alpha}(\rho, 0) & =-\ln \left(1-\frac{4|1-\rho|}{\pi^{2}}+o(|1-\rho|)\right) \\
& =\frac{4|1-\rho|}{\pi^{2}}+o(|1-\rho|)
\end{aligned}
$$

Similar calculations, in the case $\rho=1$ and $\lambda \neq 0$, and for the model studied by Kohmoto give

$$
\begin{array}{r}
\delta_{\alpha}(1, \lambda)=\frac{4|\lambda|}{\pi^{2}}+o(|\lambda|) \\
\delta_{\alpha}(\lambda)=\frac{4|\lambda|}{\pi^{2}}+o(|\lambda|)
\end{array}
$$

The most striking result is that to the first order, our result does not depend on the irrational number $\alpha$ we were starting from. The expressions (21)-(21'), are in perfect agreement with numerical studies for the Fibonacci chain or the «Octonacci » chain which is related to $\sqrt{2}-1$. For example, at $\lambda=0$ and $1-\rho=0.005$, we find $\delta=0.005 \times 0.4117 \pm 1$ and for $1-\rho=0.01$, we find $\delta=0.01 \times 0.4180 \pm 1$. Moreover, it can be shown that if $g(x)=$ $a x+b x^{2}+o\left(x^{2}\right)$ then we have

$$
a=g^{\prime}(0)=\frac{g\left(x_{1}\right) x_{2}^{2}-g\left(x_{2}\right) x_{1}^{2}}{x_{1} x_{2}^{2}-x_{2} x_{1}^{2}}+O\left(x_{1}^{2}, x_{2}^{2}\right)
$$

Here, for $x_{1}=0.005$ and $x_{2}=0.01$, the slope at the origin for $\delta_{\alpha}(\rho, 0)$ is found to be approximately 0.4054 , which must be compared to $\frac{4}{\pi^{2}}=0.40528 \ldots$ We end this section, by giving an heuristic argument for the form of $\delta_{\alpha}(\rho, 0)$. Since for $\rho=0, \rho_{\alpha}(\rho, 0)$ is infinite, we add a term of the second order in the first equality (21), so that the argument of the $\log$ is 
zero when $\rho=0$. We obtain the following «phenomenological » form for $\delta_{\alpha}(\rho, 0)$

$$
\delta_{\alpha}(\rho, 0)=-\ln \left[1-\frac{4}{\pi^{2}}|1-\rho|-\left(1-\frac{4}{\pi^{2}}\right)|1-\rho|^{2}\right] .
$$

We can compare (22) with numerical datas for $\alpha=\sqrt{2}-1$. It appears that the agreement is very good for $|1-\rho| \leqslant 0.5$ and remain good for higher values (Fig. 6). We found identical results for $\alpha=\sqrt{2}-1$ and $\alpha=(\sqrt{5}-1) / 2$, which suggests that $\delta_{\alpha}(\rho, 0)$ does not depend on $\alpha$ at all orders, at least for typical irrational numbers as those studied numerically.

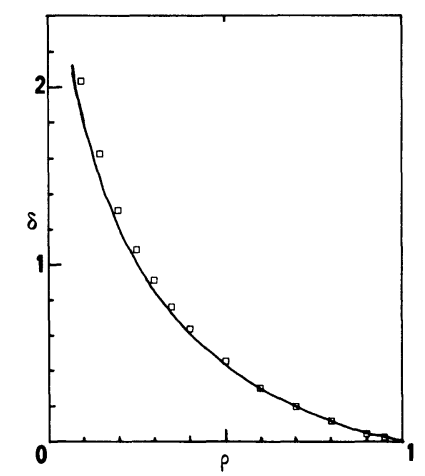

Fig. 6. - Numerical calculations for $\delta_{\alpha}(\rho, 0)$ (squares) and the phenomenological curve (22).

\section{Conclusion.}

In this paper, the most general quasiperiodic linear chain was considered, as a perturbed periodic chain. The energies for the which a gap opens belong to a dense set $\left\{ \pm 2 \cos \left(\pi j \frac{\alpha}{1+\alpha}\right) j \geqslant 1\right\}$. Hence, the gaps are labelled by only one integer, according to their intensities. The biggest ones are well described even for a quite large perturbation, while the $j$-th gap, for large $j$, whose intensity is of order $j^{-1}$ is correctly given by our treatment, if the matrix elements of the perturbative potential are quite smaller than $j^{-1}$. This treatment can be used for the infinite quasiperiodic chain as well as for finite approximant crystals of any size. Moreover, one can derive to the first order the exponant which describes the way the total band width vanishes when $n$ (the size of an approximant of the $\alpha$-quasiperiodic chain), goes to infinity. A surprising result is that, to the first order, $\delta_{\alpha}$ does not depend on $\alpha$. Numerical datas are in very good agreement with these results.

\section{Acknowledgments.}

We are very indebted to D. DiVincenzo for a critical reading of the manuscript, and to J. Bellissard for fruitfull discussions. 


\section{References}

[1] Aubry S. and André G., Ann. Israel Phys. Soc. 3 (1980) 133.

[2] Levitov S., J. Phys. France 50 (1989) 707.

[3] Mosseri R., Universality in condensed matter, Les Houches March 88, Eds. R. Jullien, L. Peliti, R. Rammal and N. Boccara (Springer) 1988, 9.

[4] Duneau M., Katz A., Phys. Rev. Lett. 54 (1985) 2688 ;

Elser V., Acta Cryst., A 42 (1988) 36 ;

Kalugin P. A., Kitaev A. Y., Levitov L. S., J. Phys. Lett. France 46 (1985) L601.

[5] Kohmoto M., Kadanoff L. P., Tang C., Phys. Rev. Lett. 50 (1983) 1870 ;

Ostlund S., Pandit R., Rand D., Schellnhuber H. J. and Siggia E. D., Phys. Rev. Lett. 50 (1983) 1873.

[6] BellissaRd J. (C.N.R.S.-Luminy) private communication.

[7] Kalugin P. A., Kitaev A. Y., Levitov L. S., Sov. Phys. JETP 64 (1986) 410 ;

Bellissard J., Statistical Mechanics and Field Theory, Mathematical Aspects, Eds. T. C. Dorlas, M. N. Hugenholtz, M. Winnink, Lect. Note Phys. 257 (1986) 99.

[8] SiRE C., Europhys. Lett., to appear.

[9] Luck J. M., Phys. Rev. B 39 (1989) 5834.

[10] Bellissard J., Iochum B., Scoppola E., Testard D., to appear in Commun. Math. Phys. 